[1]

${ }^{1}$ Dept of Pharmacology and Toxicology, Maastricht University, Maastricht, The Netherlands. ${ }^{2}$ Respiratory and Sleep Diagnostics, Midland Regional Hospital, Mullingar, Ireland. ${ }^{3}$ Dept of Pneumology and Critical Care Medicine, Thoraxklinik, University Hospital Heidelberg, Heidelberg, Germany. ${ }^{4}$ Dept of Respiratory Medicine, King's College Hospital, London, UK. ${ }^{5}$ Dept of Respiratory Medicine and Allergy, King's College London, London, UK. ${ }^{6}$ Dept of Pulmonology, Semmelweis University, Budapest, Hungary. ${ }^{7}$ Dept of Clinical Science, University of Bergen, Bergen, Norway. ${ }^{8}$ Dept of Pneumology, School of Medicine in Katowice, Medical University of Silesia, Katowice, Poland.

\title{
LSC 2016: from system approaches in lung disease to getting the job you want
}

\section{Juniors' voice}

The Lung Science Conference (LSC) 2016 generated, as in previous years, fruitful discussions and exciting networking opportunities in the breathtaking venue of the Palace Hotel, Estoril, Portugal.

\section{Systems medicine in lung disease}

The morning of Friday, March 11, 2016, started with an interesting session exploring whether systems medicine could be the answer to the unmet needs in various lung diseases, including asthma, allergic airway inflammation and chronic obstructive pulmonary disease (COPD). Peter Sterk kicked off by explaining the approach of the U-BIOPRED (Unbiased BIOmarkers in PREDiction of respiratory disease outcomes) project, in which transcriptomics, proteomics, lipidomics, metabolomics and microbiome analysis are all combined in order to obtain valuable insights into the clinical phenotypes of severe asthma [1]. This approach has already shown that integration of biomarker profiles will enable prediction of clinical course and that there is an association between single cell markers and proteomics [2]. Consequently, the clinical description of severe asthma can only be adequately described by an array of newly discovered biological entities that at the same time hold the key to the identification of critical molecular pathways as biomarkers and targets in precision medicine strategies [3]. The subsequent two oral presentations discussed other aspects affecting the clinical presentation of asthma, including the role of altered microRNA secretion via extracellular vesicles and of the eicosanoid pathway. The session was closed by Rosa Faner, who emphasised that disease heterogeneity hampers precision medicine. She stated that the first step towards solving this problem is studying the interaction between the environmental, clinical, biological and genetic networks in order to define the clinical and molecular diseasome of patients with COPD [4, 5].

\section{Methodological advances}

The session "Methodological advances as enablers" started with Yan Xu aiming to close the gap between
Cite as: Boots A, Flood $\mathrm{E}$, Kahn N, et al. Juniors' voice: LSC 2016: from system approaches in lung disease to getting the job you want. Breathe 2016; 12: 169-173. 
scientists and statisticians by showing how single cell analysis can be used to study different lung developmental stages. To this end, SINCERA has been developed, a computational pipeline for SINgle CEll RNA-seq profiling Analysis, which enables investigators to identify driving forces for each cell type or lung disease based on single cell RNA sequencing data analysis [6]. The following two oral presentations further emphasised the role of specific mediators driving lung diseases by discussing the role of extracellular vesicles in pulmonary fibrosis and of vitamin $D$ in acute respiratory distress syndrome (ARDS). Indeed, it was shown that disease-specific protein-containing extracellular vesicles are increased in pulmonary fibrosis and contribute to impaired cell-cell communication via profibrotic WNT5A signalling. Additionally, it was discussed that vitamin D has therapeutic potential mediated by resolution of neutrophilic inflammation in ARDS. The session was closed by Bertrand De Meulder, who underlined the message delivered by earlier speakers that multiple data platforms have to be used in order to define clinical phenotypes [7].

\section{Young investigator session}

This year's young investigator session (a competition for the William MacNee Award) covered a wide array of pulmonary diseases from pulmonary arterial hypertension (PAH) to idiopathic pulmonary fibrosis (IPF) and from lung cancer to COPD and asthma. Transcriptomic analysis, involving microarrays and next-generation sequencing (NGS) techniques in order to unveil and better understand the disease pathways, was discussed by the majority of the presenters, underlining the rising importance of these tools in basic and translational science.

Prakash Chelladurai presented his work on employing NGS approaches to identify disease-specific epigenetic signatures in pulmonary artery adventitial fibroblasts of donors with idiopathic $\mathrm{PAH}$, which might help to identify treatable pathways in the future.

The study presented by Isis E. Fernandez demonstrated for the first time the presence of myeloid-derived suppressor cells (MDSCs) in peripheral blood in IPF patients. MDSC levels were inversely correlated with cross-sectional and longitudinal lung function decline in IPF, reflecting disease status and indicating their biomarker potential. Therefore, controlling the expansion and accumulation of MDSCs, or blocking their immune-suppressive functions, could represent a promising novel therapeutic in IPF patients.

Sinha Anirban employed gas chromatography and mass spectrometry techniques to differentiate between baseline, exacerbations and recovery phases in the volatile organic compounds (VOCs) exhaled by moderate-to-severe asthmatics. Although the source of the VOCs isn't yet clear, he was able to employ them to differentiate between baseline and exacerbations, concluding that breathomics may be used for monitoring asthma in the future.

Kenneth Bolger presented his prospective study on patients with acute exacerbations of COPD. Using bronchoalveolar lavage and blood samples, he analysed the transcriptome with microarrays in order to better understand the innate/adaptive immune response and heterogeneity of COPD exacerbation phenotypes.

Finally, Eva Maria Garrido-Martin, who was awarded the William MacNee Award later during the conference dinner, presented her work on the tumour microenvironment and the immune infiltration in tissue isolated from human nonsmall cell lung cancer patients. Her work aimed to identify the roles of tumour-infiltrating lymphocytes and tumour-associated macrophages by analysing the transcriptome.

Overall, this exciting session introduced longer periods of active discussions between the audience and the presenting scientists, thereby enabling a vivid exchange of opinions on the cutting-edge science that was presented.

\section{Experimental models of disease}

The session entitled "Experimental models of disease" was really exceptional in terms of LSC traditions. This session on experimental approaches opened with the presentation "Natural experiments and large databases", given by Jørgen Vestbo, which represented a shift from the usual LSC "cell or rodent models" to an observational statistical model built on the basis of three merged patient populations. The model was used to explain the most important concerns in treating COPD patients, i.e. forced expiratory volume in $1 \mathrm{~s}$ (FEV1) decline and disease exacerbations. It was fascinating to see a model that could explain two major and very different pathways that take place in COPD development: 1) normal lungs with fast FEV 1 decline, and 2) development of small lungs with slow or normal FEV 1 decline [8]. Another important finding was a nested subgroup analysis showing that statins in COPD patients can effectively reduce COPD exacerbation risk, but only in patients with higher C-reactive protein levels and significant cardiovascular comorbidity. At the end of the session, Jason Bates gave a fascinating presentation on how a combination of "inverse modelling" and "forward modelling" of the whole lung in mouse models of asthma and lung injury is able to merge the structure and function in a much more detailed way than traditional approaches, concentrated on muscle 
constriction and/or allergy-related bronchial wall thickening, are capable of.

\section{Asthma and allergy}

In the "Asthma and allergy" session, Irina Lehmann gave an interesting speech on epigenetics in asthma ontology. Epigenetics are mechanisms that alter gene expression or a phenotype without altering the DNA nucleotide sequence, including (de)methylation and (de)acetylation. She highlighted the fact that smoking and exposure to different inorganic materials during pregnancy may increase the risk of asthma development in the offspring, which was indicated by hypermethylation of the child's DNA. However, it was also noted that, in general, over $80 \%$ of DNA methylation can be explained by genetic background, with only a smaller environmental influence.

In her original research presentation, Rocio Martinez-Nunez described a potential translational defect in severe asthma. Her presentation highlighted the importance of investigating ribosomal RNA, as previous transcriptomics studies failed to explain the reason for corticosteroid insensitivity in severe asthma.

On a very different topic, Lida Gharibvand reported the results of a nationwide project on the effect of particles with a $50 \%$ cut-off aerodynamic diameter of $2.5 \mu \mathrm{m}$ (PM2.5) and ozone in lung cancer development. The results clearly showed an association between PM2.5 exposure and lung cancer incidence, independent of smoking habits. However, ozone did not have a major effect. During the final presentation, the importance of environmental epidemiology on disease development was confirmed by Valérie Siroux.

\section{Posters}

The Friday poster session included 37 posters and generated fruitful discussions and exciting networking opportunities. Four chaired groups included $\sim 10$ posters each, therefore allowing ample time for poster presentations and constructive feedback in an encouragingly friendly yet productive atmosphere.

Interesting highlights included the work presented by Antonia Marazioti et al., who reported exciting data regarding the relationship between mutant KRAS and NF- $\kappa B$ activation in pleural tumour cells, which could lead to the development of malignant pleural effusions (MPEs). Mutant KRAS tumour cells were MPE competent and presented interleukin (IL)-1 $\beta$-induced IKB kinase $(\mathrm{IKK}) \alpha$ nuclear translocation that, quite interestingly, was lost when KRAS was silenced. Moreover, IKK $\alpha$ silencing of mutant KRAS tumours hampered their ability for MPE formation. It seems that KRAS mutant tumour cells require host IL-10 $\beta$ signalling for MPE precipitation. This was brought one step further by Malamati Vreka et al., who reported that IKK $\alpha$ is required for KRAS-mediated lung adenocarcinoma.

In the area of severe asthma, Ana FranciscoGarcia et al. identified a novel role for exosomal microRNAs in the disease. They reported that deficient loading of microRNA into exosomes can suggest a significant breakdown in cellular communication in the asthmatic airway, and this could represent a novel therapeutic target in the future that will address the difficult management of severe asthmatics.

The never-ending issue of corticosteroid responsiveness in COPD patients was addressed by Alen Faiz et al., who investigated genetic factors related to this effect. They identified FKBP5 as an important candidate that contributes to corticosteroid insensitivity in COPD patients.

\section{Epidemiology in the digital age}

The early evening talk given by Marcel Salathé presented a new kind of epidemiology rise as the digital age continues. Never before has this amount of data been shared throughout the world. This has provided not only the opportunity of using huge data-samples obtained by traditional health systems, but also patient-generated data through social media platforms, mobile apps and so on.

Entire networks can be described by obtaining data on people's movements and how people interact [9]. Two areas where the effectiveness of such an approach was proven in epidemiological research are infectious diseases and adverse drug event monitoring [10].

One example of social media that was explained for usage in this kind of research is Twitter. Everything posted on Twitter is considered public data and these postings have been assessed to watch negative and positive attitudes towards, for instance, vaccines. Tools are available to track how these attitudes are spread and the data can be matched with actual data on how many vaccines were eventually given.

Some questions from the audience arose concerning the validity and representability of patient-generated data. Would you write on Twitter if you just got slightly better from the new medications? Negative attitudes spread faster than positive. Do sceptical people tweet more?

It is, however, important to emphasise that this new kind of epidemiology is not supposed to replace the traditional datasets, rather to complement them. Traditional sources have high veracity whereas patient-generated data have high velocity and variety. It will be interesting to see how this opportunity to extract constructive data from huge 
amounts of unstructured data will be used in the coming years.

\section{Junior session: getting the job you want}

The Junior Members Committee organised a dedicated session for junior members at the LSC and this year's theme was "Getting the job you want", which included topics of preparing your CV, application letters and interview skills for industry and academia.

Paul Nicklin from Boehringer-Ingelheim presented a view from the industry perspective on what they are looking for with regard to CVs and application letters. His initial point was that junior members should "invest in your career" and "know what you want to do". He advised that you ensure you include content that adds value to your CV, i.e. tangible achievements, including skills that fit the role you are applying for, and ensure there is no repetition. You should present yourself as someone with ambition, skills and willingness to learn and not have your $\mathrm{CV}$ read like a job specification or just a log of your work. He reported that the area of job applications and recruitment is constantly evolving and certainly if you are not on Linkedln you are doing yourself a disservice, as both direct and indirect networks are a highly effective ways of applying for posts. Paul finished his session with the following tips:

- Know what you want and be good at it

- Use opportunities to make connections and networks

- Own your CV

- Be authentic at the interview stage
Oliver Eickelberg spoke next, from the academia perspective. His first point was "You can do what you want with your life, and remember you will be doing this for potentially the next 40 years". In academia, a cover letter is important when applying for posts. Therefore, carefully adapt this cover letter according to the post you are applying for and make sure it is perfect in form and content. Make sure you do not misspell or tab incorrectly as this shows you do not pay attention to detail. He suggests your cover letter should be honest, forward thinking and personal and should fit to the advert to which you are replying. In a similar vein to the first speaker, make use of your contacts and networks and follow up with connections made at conferences. Oliver's main advice when applying for posts in academia is to know the person you are applying to and personalise your letter.

Inge Vestbo described the interview process from the interviewer's perspective. The main starting point is to remember that "you never get a second chance to make a good first impression". It takes hard work to prepare for an interview: do extensive research, know the company/lab, know the hiring manager, background details and key interests, and dress appropriately. Her main advice for interviews was:

- Be authentic. Be yourself, match the position you are applying for and remember clothes/ appearance can distract so be careful.

- Practise! You can never practise enough at interview skills.

The junior session finished with an interactive session from Liz Elvidge, with her 10 main tips for a successful CV. After the presentations, there was a very interactive and lively discussion with the audience and the panel, which was continued offstage

\section{Tips for a successful CV}

1) Make sure your CV looks "local” to the department, company, lab, country, etc. You want the person reviewing your CV to recognise it as familiar.

2) Your CV should be changed specifically for the job: it should be applicable to each different job you are applying for. You should spend at least 1 day changing it for each different job advert. An experienced recruiter will spot "copy and paste" jobs immediately.

3) Get feedback: ask a professional recruiter who doesn't have an agenda or interest in your CV.

4) Your CV is an "organic document": it is a changing document and emphasis should be on the past 5 years. Keep older information only if it is really relevant.

5) Ask yourself, when reviewing your CV, "Why am I telling them this?", and if you don't know then take it out.

6) Remember the "30 s rule": a person reviewing your CV will make up their mind within $30 \mathrm{~s}$. Ensure that information is kept relevant and easily readable.

7) Research "norms" for the country or industry.

8) First/last page rule: keep significant information on the first and last pages, as these are the main pages the person reviewing your CV will look at.

9) Authenticity: it is a competitive world so do not be too modest. You don't want the person reviewing your CV to have to pull out the information so present it clear and proud.

10) Don't lie! 
over drinks. There was a good mix of junior and senior members present, highlighting that the area of writing a good CV and possessing good interview skills is for all!

\section{Personalised medicine}

The conference was closed by a session focusing on personalised medicine as the treatment of the future. Within this session, systems medicine was applied to pulmonary hypertension, asthma, COPD, interstitial lung diseases and drug discovery and development.

Soni Savai Pullamsetti presented how epigenetics represent attractive disease mechanisms underlying pulmonary hypertension. Indeed, epigenetic changes due to environmental and lifestyle factors can not only impose susceptibility to pulmonary hypertension but also explain phenotypic alterations and maintenance of disease state [11].

From epigenetics, the session moved towards metabolomics as Craig Wheelock discussed its application to the study of respiratory diseases, with an emphasis on COPD and asthma. Using various examples, e.g. the COSMIC (COPD and Seretide: a Multi-Center Intervention and Characterization) COPD cohort and the U-BIOPRED severe asthma study, particular challenges were presented and placed within the context of the future of utilising metabolomics for molecular phenotyping and stratified medicine [12].
The usefulness of analysing large datasets derived from different platforms to identify new therapeutic targets was additionally elucidated by Oliver Eickelberg. Focusing on IPF, he showed the progress that has been made during the last decade in understanding the natural history and phenotypes of IPF using systems medicine approaches. Moreover, he discussed that the next step is the translation of these novel concepts to the clinical setting, which will hopefully lead to increased survival rates and improved quality of life for IPF patients [13, 14].

The last presentation of the session and the conference was given by Lindsay Edwards, who presented an approach to modelling metabolic and signalling networks that can encompass different types of data and is suitable for recursive model refinement, i.e. constraint-based modelling. It was exemplified that constraint-based modelling can not only aid interpretation of high-throughput data but also predict drug targets as well as gene mutations likely to be favoured in a human population under specific conditions [15].

\section{Join us next year!}

Frankly speaking, the LSC scientific programme and networking opportunities provide the best means to boost your scientific career. Therefore, we would like to encourage all early career researchers to submit an abstract and join us at LSC 2017.

\section{Conflict of interest}

None declared.

\section{References}

1. Wheelock CE, Goss VM, Balgoma D, et al. Application of 'omics technologies to biomarker discovery in inflammatory lung diseases. Eur Respir J 2013; 42: 802-825.

2. Sung J, Wang Y, Chandrasekaran S, et al. Molecular signatures from omics data: from chaos to consensus. Biotechnolf 2012; 7: 946-957.

3. Agustí A, Antó JM, Auffray C, et al. Personalized respiratory medicine: exploring the horizon, addressing the issues. Am J Respir Crit Care Med 2015; 191: 391-401.

4. Diez D, Agustí A, Wheelock CE. Network analysis in the investigation of chronic respiratory diseases. From basics to application. Am J Respir Crit Care Med 2014; 190: 981-988.

5. Faner R, Agustí A. Network analysis: a way forward for understanding COPD multimorbidity. Eur Respir J 2015; 46: 591-592.

6. Guo M, Wang H, Potter SS, et al. SINCERA: a pipeline for single-cell RNA-seq profiling analysis. PLoS Comput Biol 2015; 11: e1004575.

7. Shaw DE, Sousa AR, Fowler SJ, et al. Clinical and inflammatory characteristics of the European U-BIOPRED adult severe asthma cohort. Eur Respir J 2015; 46: 1308-1321.

8. Lange P, Celli B, Agustí A, et al. Lung-function trajectories leading to chronic obstructive pulmonary disease. $N$ Engl J Med 2015; 373: 111-122.
9. Salathé M, Bengtsson L, Bodnar TJ, et al. Digital epidemiology. PLoS Comput Biol 2012; 8: e1002616.

10. Salathé $M$, Khandelwal $S$. Assessing vaccination sentiments with online social media: implications for infectious disease dynamics and control. PLoS Comput Biol 2011; 7: e1002199.

11. Kim JD, Lee A, Choi J, et al. Epigenetic modulation as a therapeutic approach for pulmonary arterial hypertension. Exp Mol Med 2015; 47: e175.

12. Adamko DJ, Nair P, Mayers I, et al. Metabolomic profiling of asthma and chronic obstructive pulmonary disease: a pilot study differentiating diseases. J Allergy Clin Immunol 2015; 136: 571-580

13. Huang Y, Ma SF, Vij R, et al. A functional genomic model for predicting prognosis in idiopathic pulmonary fibrosis. BMC Pulm Med 2015; 15: 147.

14. Studer SM, Kaminski N. Towards systems biology of human pulmonary fibrosis. Proc Am Thorac Soc 2007; 4: 85-91.

15. Edwards LM, Sigurdsson MI, Robbins PA, et al. Genome-scale methods converge on key mitochondrial genes for the survival of human cardiomyocytes in hypoxia. Circ Cardiovasc Genet 2014; 7: 407-415. 